E3S Web of Conferences 1, 31003 (2013)

DOI: $10.1051 / \mathrm{e} 3$ sconf/20130131003

(C) Owned by the authors, published by EDP Sciences, 2013

\title{
Electroleaching of Copper Waste with Recovery of Copper by Electrodialysis
}

\author{
P. Nuñez ${ }^{1}$ and H. K. Hansen ${ }^{1}$ \\ ${ }^{1}$ Departamento de Ingeniería Química y Ambiental, Universidad Técnica Federico Santa María, Avenida España 1680, \\ Valparaíso, Chile, henrik.hansen@usm.cl
}

\begin{abstract}
A new process to leach and recover copper from solid waste using electric fields was designed. The leaching with electro migration is presented as an alternative to traditional leaching. Preliminary data indicate that the copper ion migration is facilitated by using the electrical potential difference; therefore applying a potential difference in the processes of leaching facilitates the removal of copper. This is especially useful when mineral concentrations are very low. Different phenomena associated with transport of copper in solution are studied to generate a model able predict the state of the copper ion concentration in time. A kinetic model for the process was developed and fitted very well the experimental data.
\end{abstract}

Keywords: Solid waste treatment, electroleaching, copper recovery

\section{Introduction}

The recovery processes in the production of copper from oxidized copper minerals contain mainly three different unit operations (leaching, solvent extraction and electrowinning). The leaching is carried out in piles with sulfuric acid, which produces solutions with low concentrations of copper. In the solvent extraction process the copper is concentrated, achieving higher concentrations of electrolytes. Numerous studies have been done to improve the profitability of these stages of copper processing, but this profitability it is linked primarily to high grade ores.

The low grade ore such as copper mining wastes can be treated with alternative technologies of low cost. In this paper, the phenomenon of leaching in conjunction with electro migration of copper ions is studied as a real and feasible alternative for these cases. In addition, important factors are considered such as the low concentration of metal in the solid, acid leaching and a difference in electrical potential applied to the copper solutions.

The leaching mechanisms used in the mining are varied and depend on the nature of the ore, usually the heap leaching is most commonly used, given the characteristics of the ore extracted. However, this study is based on leaching by agitation, given the conditions of experimental work. This type of leaching has been enhanced with an electrodialysis system, which has a pair of electrodes and a pair of ion-selective membranes, thus by applying an electrical potential promotes the copper removal from the reactor resulting in a solution copper concentrate.

Different phenomena associated with transport of copper in solution are studied to generate a model able predict the state of the copper ion concentration in time.

\section{Methods and Materials}

In this study, the electrodialysis phenomenon is applied as an alternative method of recovery of metal ions, using ion exchange membranes. By applying an electrical potential in the copper solution, copper ions pass through the membrane to produce a concentrated copper solution.

The method used is based on the recovery of copper from copper oxide ore using a batch reactor, which can produce a solution rich in copper. Figure 1 shows the experimental setup and the reactor.

The reactor has a volume of $16 \mathrm{~L}$ in the center compartment and $1 \mathrm{~L}$ in each cell (anode and cathode), and includes ion exchange membranes, graphite electrodes and a power supply. Current work is underway with copper sulfate and copper ore in order to study phenomena associated with the electro migration of copper ions in this reactor.

Experimentally the variables evaluated in this study were:

Concentrations: It is the most important variable; it determines how effective the electro migration process is. 


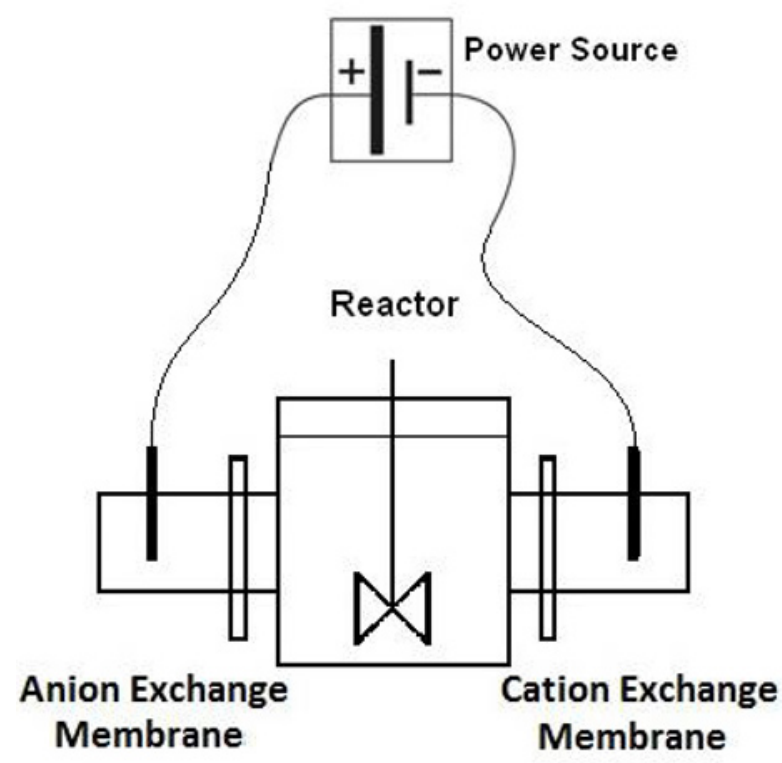

Fig.1 Experimental setup

Electrical conductivity: It has direct impact on the processes of electro migration; due to that changes in electrical conductivity generate different resistances, therefore different rates of copper migration.

Voltage: It is the driving force of the process.

Current: The current is important from the point of view that the current density actually helps the mobility of copper ions.

Particle size: It also has influence of the resistance of the overall process; remember that this is generated from the leachate to be treated.

\section{Results and Discussion}

The displacement of copper ions from one solution to another through ion exchange membranes is complex to describe; it combines three different forces in the mass transport: diffusion, electro migration and convection. The Nernst-Planck equation is used to associate this phenomenon. In equation (1) and (2) shows the Nernst-Planck equation for species $\mathrm{j}$ (for this study $\mathrm{j}$ represents the copper ions $\mathrm{Cu}^{+2}$ )

$$
J_{j}=J_{d}+J_{m}+J_{c}
$$

$$
J_{j}=-D \frac{d C_{j}}{d x}-\frac{z F D C_{j}}{R T} \frac{d \Phi}{d x}+C_{j} v
$$

In a low permeability porous medium (such as membranes) in presence of electric fields, electro migration and electro osmosis are generally considered the dominant transport mechanisms. Electro osmosis can be incorporated into the Nernst-Planck equation as shown in equation (3) where $J_{v}$ is fluid volume flow per unit area and time and $\Theta$ is a parameter of coupling between the solvent flow and the $\mathrm{j}$ ions $\left(\mathrm{Cu}^{+2}\right)$.

$J_{j}=-D \frac{d C_{j}}{d x}-\frac{z F D C_{j}}{R T} \frac{d \Phi}{d x}+C_{j} v+\Theta J_{v} C_{j}$

The developed model is based on this equation to describe the displacement of copper ions through the membrane with time, for this a series of assumptions and simplifications is necessary.

Among the considerations that the changes in voltage and concentration through the membrane are constant, these assumptions are presented in equation (4).

$J_{j}=-D \frac{\Delta C_{j}}{\Delta x}+\left(-\frac{z F D}{R T} \frac{\Delta \varphi}{\Delta x}+J_{v}+v\right) * C_{j}$

The above equation describes the mobility of ions from inside the reactor, on the other side of the membrane material balance arises in equation (5).

$\frac{d\left(V \cdot C_{j}\right)}{d t}=J_{j} \cdot A$

Replacing (4) in (5).

$\frac{d C_{j}}{d t}=\left(-\frac{A D \Delta C_{j}}{V \Delta x}\right)+\left(-\frac{A z F D \Delta \Phi}{V R T \Delta x}+v+J_{v}\right) * C_{j}$

Integrating.

$$
\int \frac{1}{\left(-\frac{A D \Delta C_{j}}{V \Delta x}\right)+\left(-\frac{A z F D \Delta \Phi}{V R T \Delta x}+J_{v}+v\right) * C_{j}} d C=\int d t
$$

$C(t)=\frac{\left(-\frac{A D \Delta C_{j}}{V \Delta x}\right)}{\left(\frac{A z D D \Delta}{V R T \Delta x}-J_{v}\right)}+\frac{E X P\left[-t *\left(\frac{A z F D \Delta \Phi}{V R T \Delta x}-v-J_{v}\right)\right]}{\left(\frac{A z F D \Delta \phi}{V R T \Delta x}-v-J_{v}\right)}$

Finally, the model that describes changes in concentration of $\mathrm{Cu}+2$ in the reactor is presented in equation (8) and represented graphically in Figure 2.

\section{Conclusion}

The use of current does increase the speed of copper ion migration, decreasing the concentrations of copper in the contaminated soil and recovering copper in concentrated solutions.

Between the factors that affect the electro migration of copper, electrical conductivity of the solution is most important. In low conductivity solutions other phenomena are present that make copper move, being irrelevant the contribution that can generate the potential 


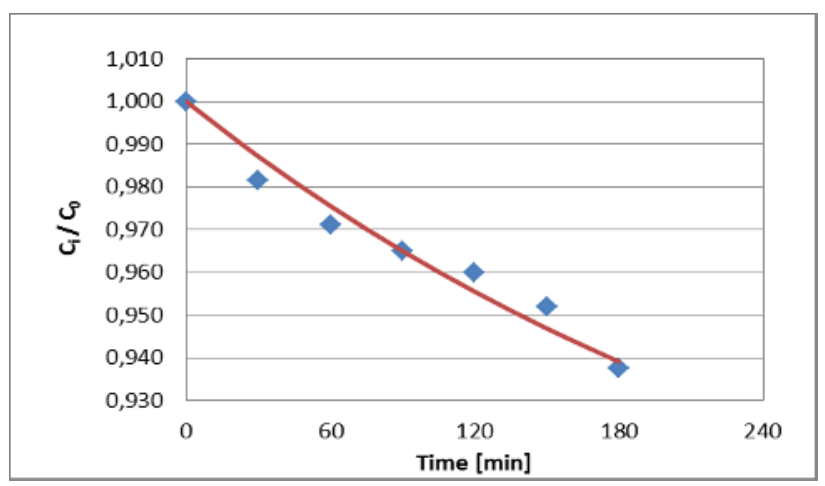

Fig.2 Model prediction vs experimental data

difference in the half. By contrast, in a solution with a high electrical conductivity, displacement of copper ions is strongly influenced by a potential difference, favoring the electro kinetic transport phenomena.

The leaching with electro migration is presented as an alternative to traditional leaching. Preliminary data indicate that the copper ion migration is facilitated by using the electrical potential difference; therefore applying a potential difference in the processes of leaching facilitates the removal of copper. This is especially useful when mineral concentrations are very low.

\section{Acknowledgements}

The authors thank the financial supports providing from Chilean FONDECYT Project N| 1120111. 